\title{
Meta-Analysis: The Efficacy and Safety of Paricalcitol for the Treatment of Secondary Hyperparathyroidism and Proteinuria in Chronic Kidney Disease
}

\author{
Tianzhao Han, ${ }^{1}$ Gong Rong, ${ }^{1}$ Dayong Quan, ${ }^{1}$ Ying Shu, ${ }^{1}$ Zhu Liang, ${ }^{1}$ Ninglan She, ${ }^{1}$ \\ Manli Liu, ${ }^{1}$ Bing Yang, ${ }^{1}$ Gong Cheng, ${ }^{1}$ Yongman Lv, ${ }^{2}$ and Leonard Stern ${ }^{3}$ \\ ${ }^{1}$ The Department of Nephrology, The Third People's Hospital of Chengdu, The Chongqing Medical University, Chengdu 610031, China \\ ${ }^{2}$ Department of Nephrology, Tongji Hospital, Tongji Medical College, Huazhong University of Science and Technology, \\ Wuhan 430000, China \\ ${ }^{3}$ Division of Nephrology, Department of Medicine, Columbia University Medical Center, 622 West 168th Street, PH 4-124, \\ New York, NY 10032, USA
}

Correspondence should be addressed to Leonard Stern; 1s38@columbia.edu

Received 4 April 2012; Revised 18 July 2012; Accepted 9 August 2012

Academic Editor: Sihe Wang

Copyright (C) 2013 Tianzhao Han et al. This is an open access article distributed under the Creative Commons Attribution License, which permits unrestricted use, distribution, and reproduction in any medium, provided the original work is properly cited.

\begin{abstract}
Introduction. Previous studies have demonstrated the safety and efficacy of using Paricalcitol for the treatment of secondary hyperparathyroidism (SHPT) in patients on dialysis. The aim of the current meta-analysis was to assess the safety and efficacy of Paricalcitol for the management of SHPT in patients with chronic kidney disease (CKD) not yet on dialysis. A secondary aim was to determine if sufficient data was available to assess the effect of Paricalcitol for the management of proteinuria. Methods. A meta-analysis was conducted using the Cochrane Collaboration's RevMan 4.2 software. Results. Paricalcitol is effective in lowering PTH in patients with CKD not yet on dialysis and is also effective in lowering proteinuria in diabetic CKD patients. However, we uncovered a safety signal identifying an elevated calcium phosphate product and a trend towards the development of hypercalcemia. A phosphate elevation was not demonstrated because the target used in the clinical studies was a $P>5.5 \mathrm{mg} / \mathrm{dl}$, a value appropriate for dialysis patients and not CKD patients. Conclusion. Although Paricalcitol is effective in lowering PTH, we advise caution in the use of any active Vitamin D analogues in patients with CKD because of the potential risk of exacerbating vascular calcification.
\end{abstract}

\section{Introduction}

One of the greatest therapeutic challenges in the chronic kidney disease population is the management of bone and mineral metabolic parameters in order to preserve bone integrity, minimize cardiovascular calcification, and manage serum levels of parathyroid hormone $(\mathrm{PTH})$, calcium and phosphorus. The cornerstone of this condition is characterized by the CKD-MBD (chronic kidney disease-mineral bone disorder) syndrome where there is secondary hyperparathyroidism (SHPT), manifested by parathyroid hyperplasia and upregulated synthesis and secretion of PTH $[1,2]$. In addition, there is an elevation of the serum phosphate, a reduction in serum calcium, and an absolute reduction of active vitamin D (calcitriol) levels caused by a reduction of the synthetic 1a-hydroxylase encoded by the CYP27B1 gene and an increase in the catabolic 24a-hydroxylase encoded by the CYP 24 gene. Both of these enzymatic changes are characteristically present in CKD and are very likely mediated by the high levels of FGF23 also characteristically present in CKD [3]. The other features of this syndrome include renal osteodystrophy where an abnormality of bone anabolism causes high bone turnover disease, fractures, vascular calcification, and cardiovascular complications. Slowing the rate of progression towards end-stage renal disease is one of the key goals of medical intervention in this patient group.

Secondary hyperparathyroidism (SHPT) is a common and early complication of CKD. Targeting SHPT in patients with CKD and end stage renal disease on dialysis with active vitamin $\mathrm{D}$ analogues such as Paricalcitol has been the 
subject of multiple research studies in patients. Numerous studies of mixed quality, targeting various surrogate outcome measures have been published clearly demonstrating the biologic importance of the therapy $[4-7,10]$. Active vitamin $\mathrm{D}$ analogues including Paricalcitol have shown demonstrably favorable effects on SHPT [4, 6-11] and proteinuria $[4-6,12-$ 14]. In these studies, no clinically important or statistically evident change in eGFR has been shown $[4-6,8,9,12$, 14]. The active vitamin $D$ analogues have also shown clear evidence for decreases in cardiovascular events [15], and improved survival in hemodialysis patients. [16, 17]. The majority of the published research data, however, has been obtained in patients on dialysis [7, 10, 11, 15-17]. The role of treatment with Paricalcitol in CKD targeting early SHPT is less clear [4-6, 8, 9]. A previously published meta-analysis summarized the efficacy of Paricalcitol therapy for chronic kidney disease combining the data for patients receiving and not yet on dialysis and concluded that Paricalcitol suppresses iPTH and lowers proteinuria in patients with stages $2-5 \mathrm{CKD}$ without an increased risk of adverse events [14]. At least one study has presented evidence for the use of vitamin $\mathrm{D}$ analogues in the prevention of vascular calcification [18].

One of the many biologic actions of active vitamin $\mathrm{D}$ is to cause an increase in the amount of intestinal calcium and phosphorus absorption, resulting in hypercalcemia and hyperphosphatemia. At higher than physiologic dosages, active vitamin D may actually increase bone resorption. Paricalcitol, a synthetic vitamin D analogue engineered to effectively suppress secretion of PTH with fewer hypercalcemic and hyperphosphatemic side effects, has been shown to effectively reduce $\mathrm{PTH}$ and also reduce proteinuria in recent studies in patients with CKD $[4-6,12,14,16,17]$. The goal of the present meta-analysis was to evaluate the efficacy and safety of treatment with Paricalcitol in the management of SHPT, proteinuria, and preservation of renal function in patients with CKD. In particular, we wanted to evaluate whether there is sufficient published data to recommend treatment with Paricalcitol to patients with CKD and SHPT not yet on dialysis.

\section{Methods}

2.1. Data Sources and Searches. The literature searches for randomized, controlled trials (RCTs) of Paricalcitol in CKD were retrieved from PubMed, Medline, EMBASE, Elsevier Science, Karger, Free Medical Journals, BMJ, Nature and CNKI between 1993 and 2009 by using the search strategy "Paricalcitol Limits Activated: Humans, Randomized Controlled Trial." The Reference sections of included articles were reviewed for other potentially relevant citations. Finally, the authors of included studies were personally contacted to obtain further information.

\subsection{Study Selection}

2.2.1. Inclusion Criteria. Only randomized, controlled trials were considered for inclusion in this analysis. Other criteria included the following. (1) Treatment group received Paricalcitol and the control group received placebo. (2) Definitions of proteinuria, hypercalcemia and hyperphosphatemia were similar in all reports. (3) Each study had the inclusion/exclusion criteria and participants were considered eligible. The authors must have given the size of their samples, a significance level, and their 95\% confidence intervals (CIs). The methods of analysis using analysis of covariance or Fisher's exact test were statistically acceptable. (4) Studies of other vitamin D compounds or in other non-CKD disease states were excluded.

2.2.2. Exclusion Criteria. Patients were excluded if they failed to meet the inclusion criteria or if they failed to complete the study protocol. We also excluded animal studies.

\subsubsection{Efficacy Indices}

Change in iPTH. Defined as achieving a greater than or equal to $30 \%$ decrease in $\mathrm{iPTH}$ from baseline for two consecutive measures.

Proteinuria. Defined as a statistically significant decrease in urinary protein-creatinine ratio or urinary albumincreatinine ratio.

$\triangle e G F R$. The mean change in eGFR from baseline to final visit. Hypercalcemia. Defined as two consecutive calcium measurements of greater than $2.62 \mathrm{mmol} / \mathrm{L}$ or $10.5 \mathrm{mg} / \mathrm{dL}$.

Hyperphosphatemia. Defined as two consecutive phosphorus measurements of greater than $5.5 \mathrm{mg} / \mathrm{dL}$.

Elevation in Calcium $\times$ Phosphorus Product. Defined as two consecutive calcium $\times$ phosphate product values of greater than $55 \mathrm{mg}^{2} / \mathrm{dL}^{2}$.

2.3. Data Extraction and Quality Assessment. Three independent authors extracted relevant data from eligible studies. Discrepancies were resolved by discussion and by referencing the original report. Two independent authors assessed each trial using the Jadad rating scale [19] and referred to the Cochrane Reviewers' Handbook 4.2.6 about the quality of randomized controlled trials (randomization, blinding, withdrawal and loss, allocation concealment, and intentional Analysis-A: adequate, B: unclear, C: inadequate, and D: not used) [20].

2.4. Data Synthesis and Analysis. A meta-analysis was conducted using the Cochrane Collaboration's RevMan 4.2 software. A test of heterogeneity was assessed by the chisquare test $\left(P\right.$ value and $I^{2}$ ), which describes the percentage of variability in the effect and estimates the contribution of heterogeneity rather than by chance [21, 22]. We summarized treatment effects as relative risks (RRs) for categorical variables and weighted mean differences for continuous variables, with 95\% CIs. If no heterogeneity existed among studies $\left(P \geq 0.05\right.$ and $\left.I^{2}>0.5\right)$, the fixed effect model was used. An $I^{2}=0$, indicated that the variation was caused by sampling error; A $I^{2}<0.25$, indicated a slight degree of heterogeneity; A $I^{2}>0.25-<0.5$, indicated a moderate degree of heterogeneity; A $I^{2}>0.5$, indicated a high degree of 
heterogeneity [23]. If $I^{2}>0.5$ or $P<0.05$, the heterogeneity among these studies was considered statistically significant and a descriptive analysis was employed.

\section{Results}

A total of 25 articles were retrieved in the initial search. We found 1 ongoing study (VITAL study) [23] and fortunately obtained results about the published VITAL study from the corresponding author [12]. Examination of the abstracts and full texts allowed us to exclude non case-control studies or studies where the participants did not have CKD, leaving 9 articles that form the basis of this meta-analysis.

3.1. Trial Characteristics. The 9 studies included a total of 1113 participants; 20 participants did not complete the protocol and are excluded leaving 1093 participants included in this meta-analysis. $58.2 \%$ had diabetic kidney disease, $20.6 \%$ had nondiabetic kidney disease, and the remainders were not characterized. The characteristics of the nine studies and the efficacy parameters are summarized in Tables 1(a) and $1(\mathrm{~b})$, respectively.

3.2. Trial Quality. We assessed the quality of included studies using the Jadad rating scale [19] and referred to the Cochrane Reviewer's Handbook 4.2.6 for guidelines used to rate the quality of randomized controlled trials [20] (Table 2). The main factors influencing quality were allocation concealment, intentional analysis, withdrawal, and dropout. The primary reasons described for premature withdrawal of these patients were kidney transplantation, increase in iPTH levels, unblinding, and failure to complete a scheduled protocol visit. Each study received a grade of A or B. The Jadad rating score was assigned from 2 to 5 points.

\subsection{Meta-Analysis Results}

3.3.1. Two Consecutive Decreases of Greater Than or Equal to $30 \%$ in $i P T H$. The six studies that compared this efficacy index included a total of 720 participants; 369 and 351 treated with Paricalcitol and placebo, respectively (Figure 1). All six studies had homogeneity (heterozygosity test, $\left.\chi^{2}=3.28, P=0.77, I^{2}=0 \%\right)$. When the fixed-effect model was used to merge RR values, the pooled RR was 6.97 (95\% CI 5.27-9.23, $Z=13.57, P<0.00001$; Table 3). This indicated that the Paricalcitol treated patients had a statistically significant sustained reduction in serum iPTH levels during the observation period.

3.3.2. $\triangle e G F R$. Among the three studies that reported this efficacy index, a total of 468 patients were included; 221 and 247 in the Paricalcitol and placebo groups, respectively (Figure 2). All three studies had heterogeneity (heterozygosity test, $\chi^{2}=240.01, P<0.00001, I^{2}=98.8 \%$; Table 3). Because of this high heterogeneity, the effect of Paricalcitol on the $\triangle \mathrm{eGFR}$ using formal meta-analysis technique is not certain. Using a more conventional descriptive analysis, the data from each study (Table 4) show no statistically significant difference between the Paricalcitol-treated and placebo groups implying that Paricalcitol had no negative impact on renal function.

3.3.3. Proteinuria. Three studies included this efficacy index with a total of 349 participants; 227 and 122 in the Paricalcitol and placebo groups, respectively (Figure 3 ). The majority $(88.6 \%)$ had diabetic kidney disease. All three studies had homogeneity (heterozygosity test, $\chi^{2}=3.72, P=0.16, I^{2}=$ $46.2 \%)$. When the fixed-effect model was used to merge RR values, the pooled RR was 1.57 (95\% CI 1.20-2.04, $Z=3.29$, $P=0.0010$; Table 3). This indicated that Paricalcitol-treated patients with diabetic CKD had a statistically significant reduction in proteinuria compared to placebo.

Two studies, with 199 participants reported the effect of Paricalcitol with varying dosages. 99 patients received 1 microgram and 100 received a 2 microgram dose (Figure 4). Both studies had homogeneity (heterozygosity test, $\chi^{2}=$ 0.48, $\left.P=0.49, I^{2}=0 \%\right)$. When the fixed-effect model was used to merge RR values, the pooled RR was 1.04 (95\% CI $0.81-1.33, Z=0.32, P=0.75$; Figure 4). Comparing the 1 and 2 microgram Paricalcitol-treated groups; there was no statistically significant difference in proteinuria reduction.

3.3.4. Hypercalcemia. Among the six studies where this efficacy parameter is reported, 875 participants were evaluated for the incidence of hypercalcemia; 495 and 380 in Paricalcitol and placebo groups, respectively (Figure 5). All six studies had homogeneity (heterozygosity test, $\chi^{2}=$ $\left.0.64, P=0.96, I^{2}=0 \%\right)$. When the fixed-effect model was used to merge RR values, the pooled RR was 2.91 (95\% CI 0.86-9.90, $Z=1.71, P=0.09$; Table 3 ). This indicated that there was no statistically significant difference in the incidence of hypercalcemia between the Paricalcitol and placebo groups though a trend towards hypercalcemia was evident in the Paricalcitol-treated groups, where 10 of 495 in the Paricalcitol group and 1 of 380 in the placebo group developed hypercalcemia. There was insufficient data to determine a dose-response effect comparing 1 ug versus 2 ug dosing.

3.3.5. Hyperphosphatemia. Among the three studies reporting this efficacy parameter, 478 participants were evaluated for the incidence of hyperphosphatemia; 233 and 245 in the Paricalcitol and placebo groups, respectively (Figure 6). All three studies had homogeneity (heterozygosity test, $\chi^{2}=$ 0.60, $\left.P=0.90, I^{2}=0 \%\right)$. When the fixed-effect model was used to merge RR values, the pooled RR was 0.94 (95\% CI 0.56-1.58, $Z=0.22, P=0.82$; Table 3). This indicated that there was no statistically significant difference in the incidence of hyperphosphatemia between the Paricalcitol and placebo groups. The studies included, used $5.5 \mathrm{mg} / \mathrm{dL}$, as the upper limit of the acceptable range for the serum phosphate. The current KDIGO guidelines recommend targeting the serum phosphate to the normal range, in this case a level $<4.0 \mathrm{mg} / \mathrm{dL}$ [24]. 

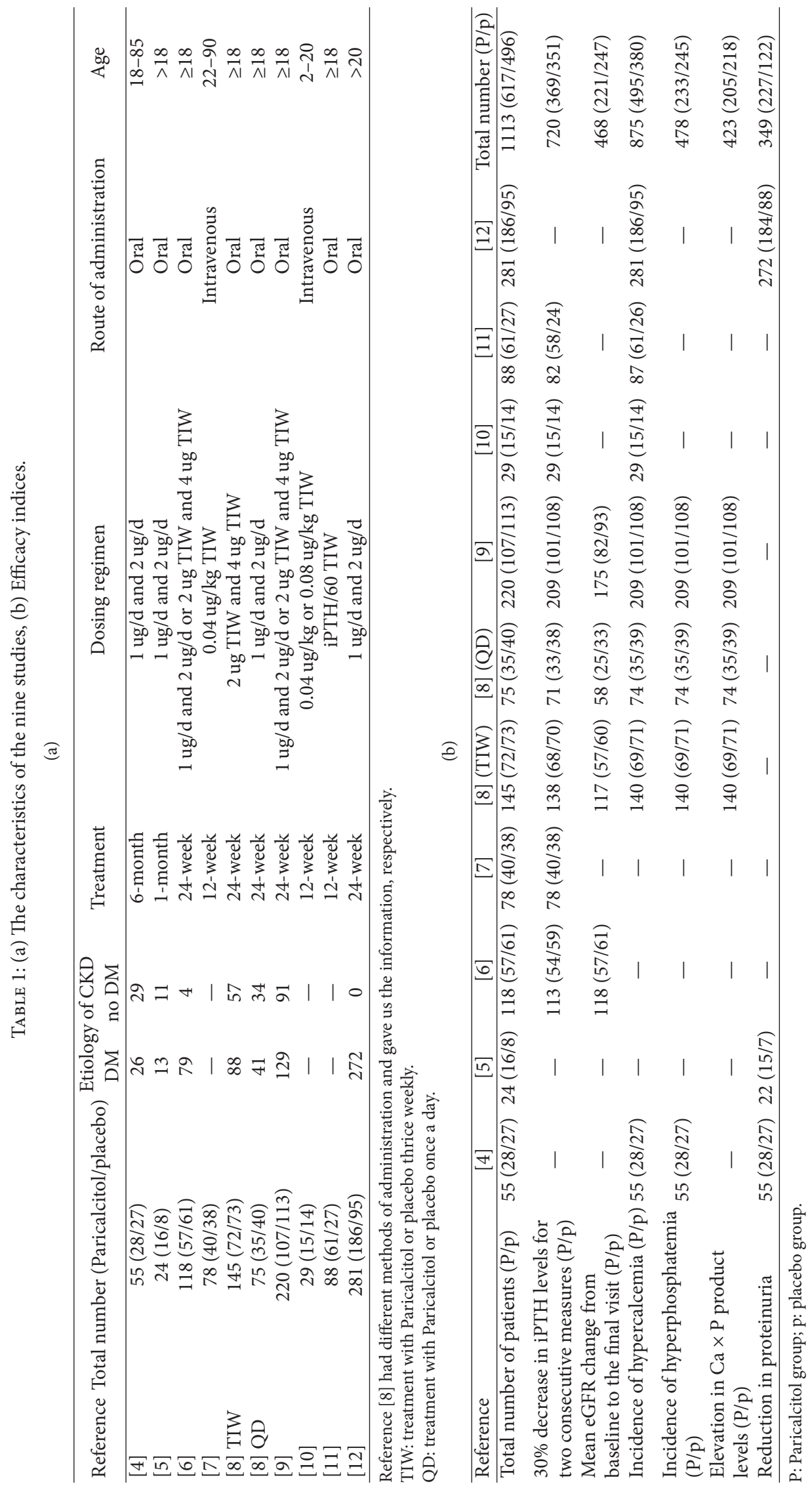


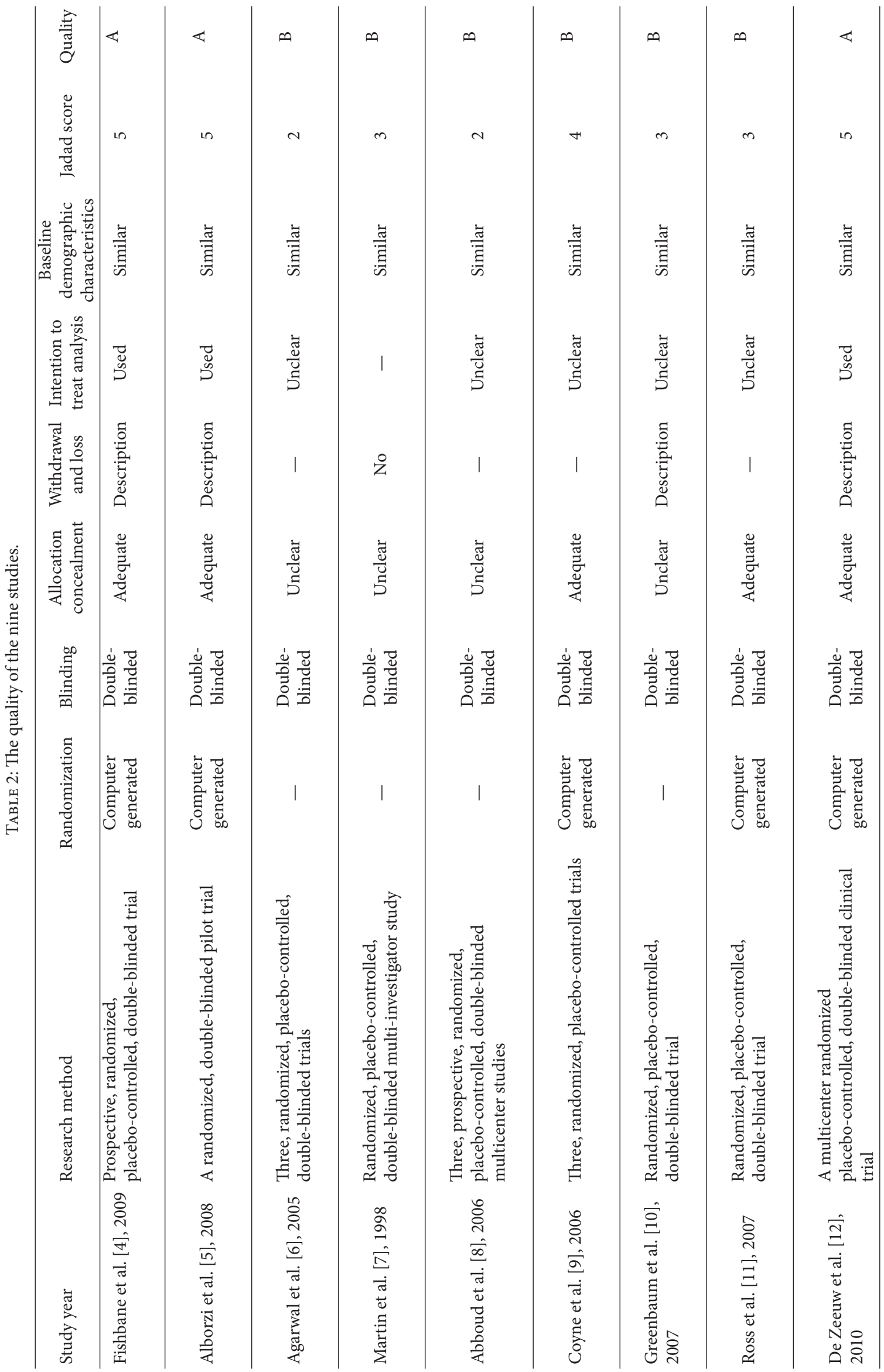




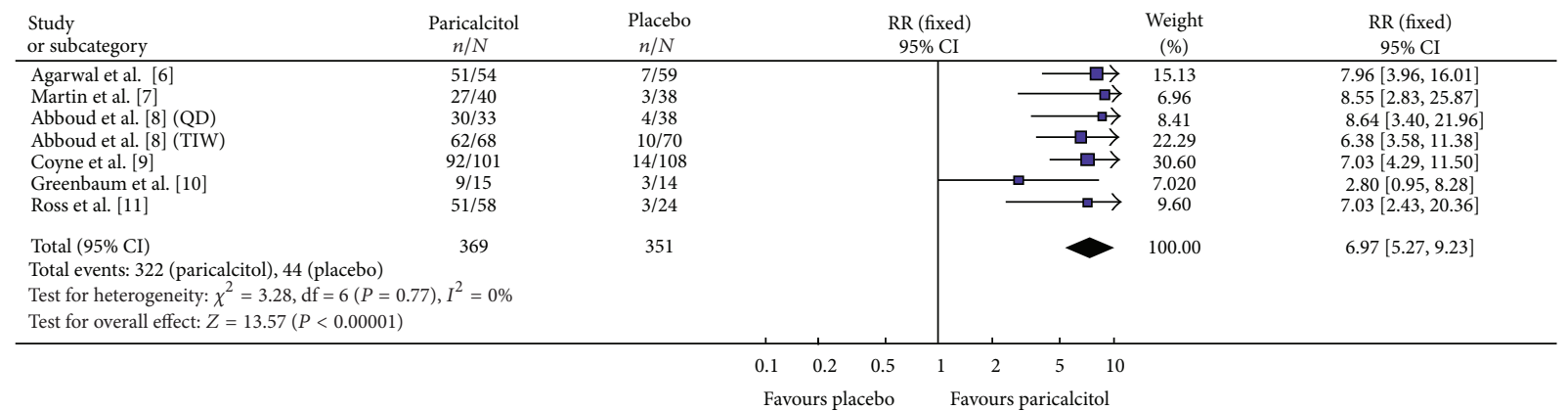

FIGURE 1: Comparison of the probability of achieving $\geq 30 \%$ decrease in iPTH from baseline for two consecutive measures.

TABLE 3: Meta-analysis.

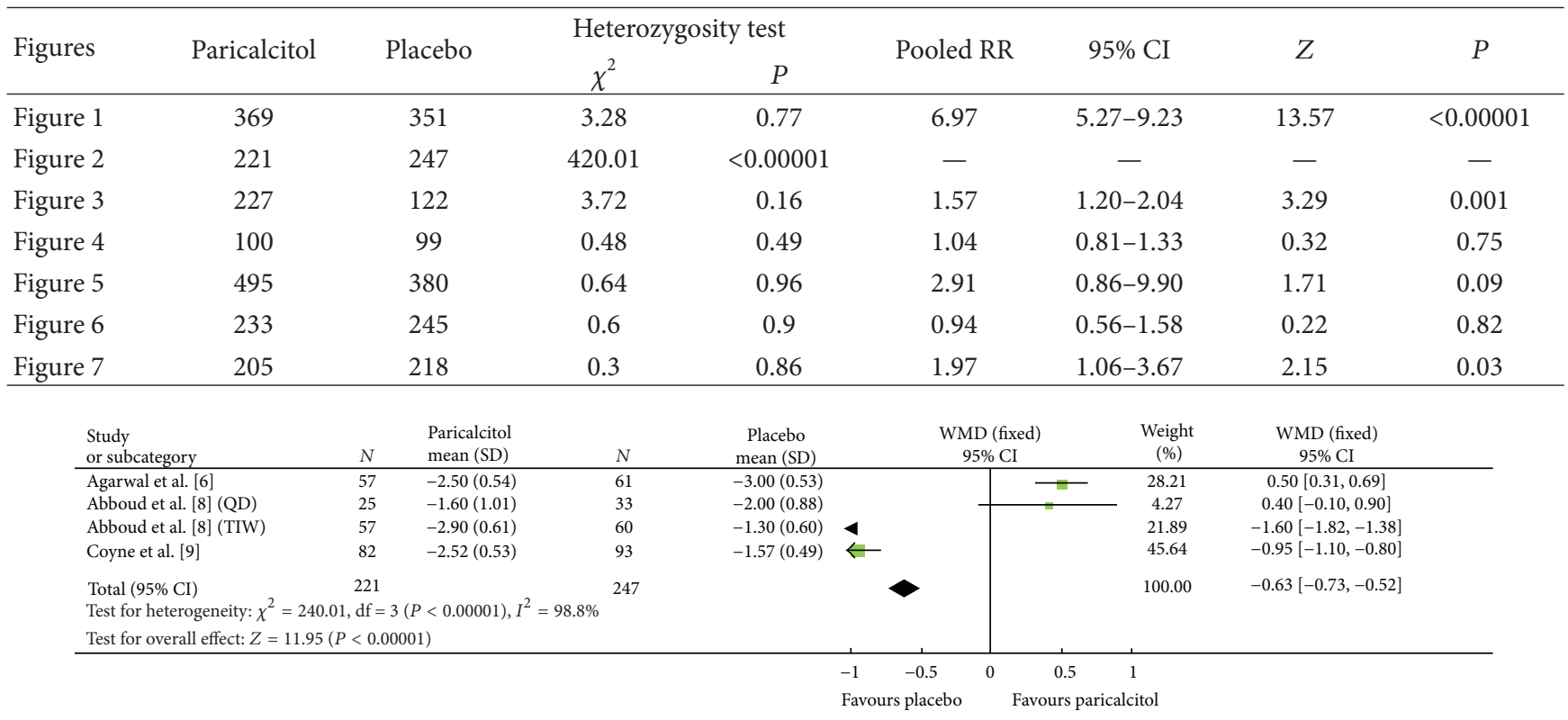

FIGURE 2: Comparison of the mean change in eGFR $\left(\mathrm{mL} / \mathrm{min} / 1.73 \mathrm{~m}^{2}\right)$ from baseline to final visit.

TABLE 4: Comparison of the mean change in eGFR from baseline to final visit ( $\triangle \mathrm{eGFR}$ ).

\begin{tabular}{lccc}
\hline \multirow{2}{*}{ Studies } & \multicolumn{2}{c}{$\Delta \mathrm{eGFR}\left(\mathrm{mL} / \mathrm{min} / 1.73 \mathrm{~m}^{2}\right) \pm \mathrm{SD}$} & $P$ value \\
& Paricalcitol group & Placebo group & $P=0.51$ \\
Agarwal et al. [6] & $-2.5 \pm 0.54$ & $-3.0 \pm 0.53$ & $-1.3 \pm 0.60$ \\
Abboud et al. [8] (TIW) & $-2.9 \pm 0.61$ & $-2.0 \pm 0.88$ & $P=0.066$ \\
Abboud et al. [8] (QD) & $-1.6 \pm 1.01$ & $-1.57 \pm 0.494$ & $P=0.78$ \\
Coyne et al. [9] & $-2.52 \pm 0.526$ & $P=0.187$ \\
\hline
\end{tabular}

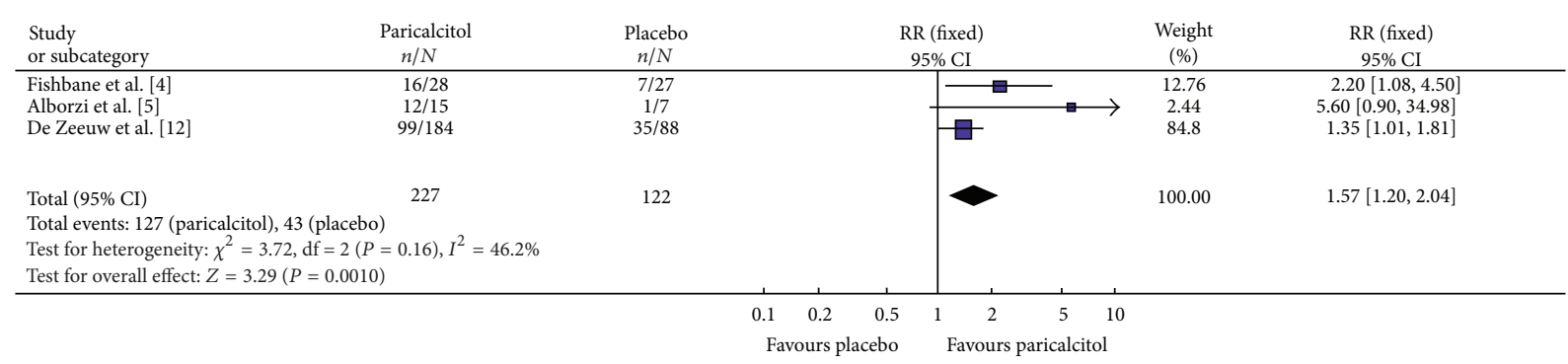

FIgURE 3: Comparison of the reduction in proteinuria. 


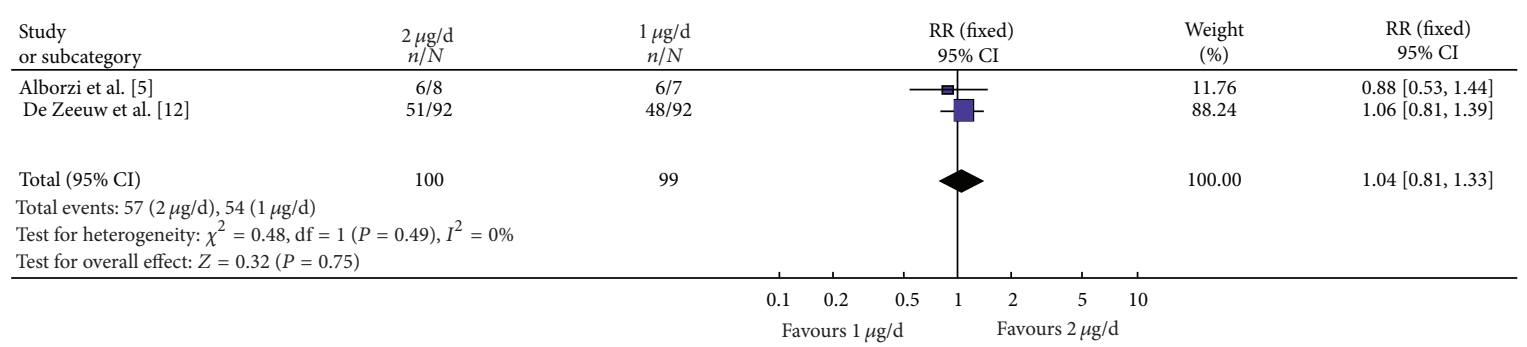

FIGURE 4: Comparison of the reduction in proteinuria netween the $1 \mathrm{ug} / \mathrm{d}$ and $2 \mathrm{ug} / \mathrm{d}$ groups.

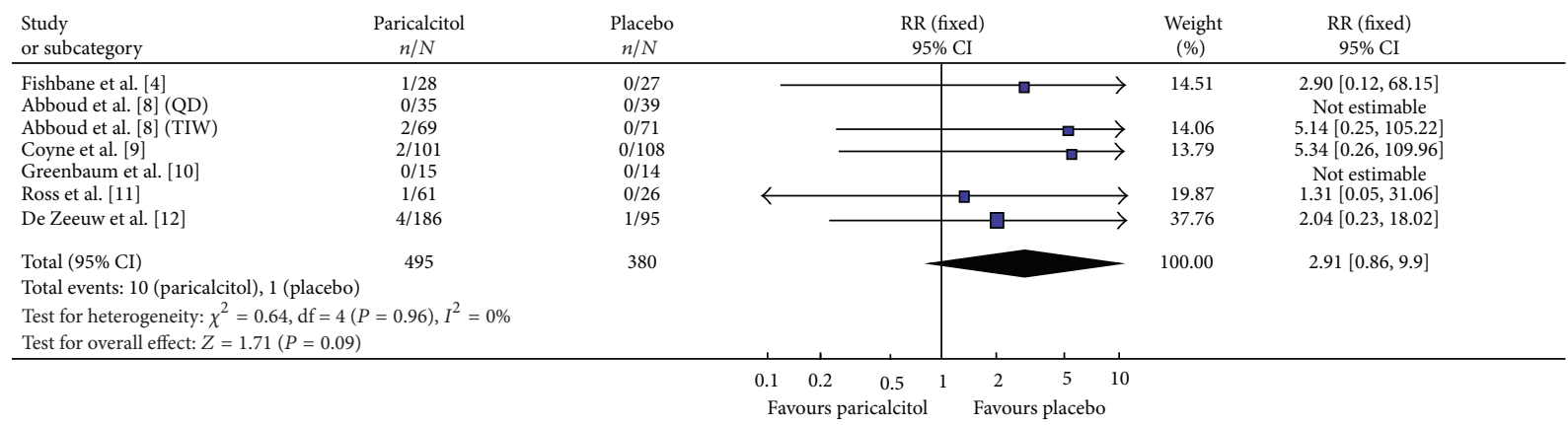

Figure 5: Incidence of hypercalcemia.

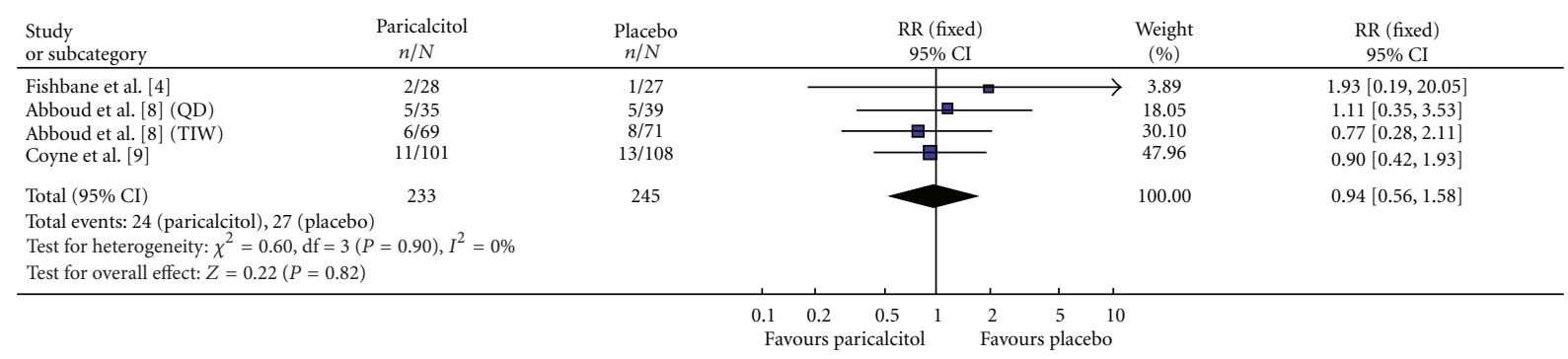

FIGURE 6: Incidence of hyperphosphatemia.

3.3.6. Elevation in Calcium $\times$ Phosphorus $(\mathrm{Ca} \times \mathrm{P})$ Product. In the two studies reporting this efficacy parameter, 423 participants were evaluated for a change in calcium $\times$ phosphorus product levels, 205 and 218 in the Paricalcitol and placebo groups, respectively (Figure 7 ). Both studies had homogeneity (heterozygosity test, $\chi^{2}=0.30, P=0.86, I^{2}=0 \%$ ). When the fixed-effect model was used to merge RR values, the pooled RR was 1.97 (95\% CI 1.06-3.67, $Z=2.15, P=0.03$; Table 3 ). While, the data shows that there was no statistically significant difference in the incidence of an elevation in $\mathrm{Ca}$ $\times \mathrm{P}$ product between the Paricalcitol and placebo groups for each individual study (Table 5), the pooled data in the meta-analysis do show a statistically significant increase in the incidence of an elevated $\mathrm{Ca} \times \mathrm{P}$ product between the Paricalcitol- and placebo-treated groups $(P=0.03)$.

\section{Discussion}

The CKD-MBD syndrome characteristic of chronic kidney disease (CKD) of virtually any etiology imposes the burden of excess mineral retention enhancing cardiovascular risk by promoting the development of vascular calcification [13, 25-27]. The hallmark biochemical abnormalities identified in $\mathrm{CKD}$ are a reduced level of active vitamin $\mathrm{D}$ which results in an elevated blood level of PTH by upregulation of the synthesis and secretion of parathyroid hormone [27]. Targeting PTH synthesis by treating the active vitamin D insufficiency is the generally accepted standard of care [28, 29]. However, the treatment with active vitamin D analogues may promote further retention of calcium and phosphate and potentially worsen the cardiovascular risk profile of the patients with CKD being treated. Paricalcitol is a synthetic active vitamin D analog chemically designed to limit the absorption of calcium and phosphate by the intestine [30]. In low doses, Paricalcitol results in a 10 -fold reduction of calcium absorption compared to calcitriol [31]. Paricalcitol acts as an active agonist for the vitamin $\mathrm{D}$ receptor and in the parathyroid gland negatively regulates the gene transcription for PTH thus lowering the blood parathyroid hormone level [32-35]. However, Paricalcitol like all currently available active vitamin $\mathrm{D}$ analogues directly binds to the VDR in 


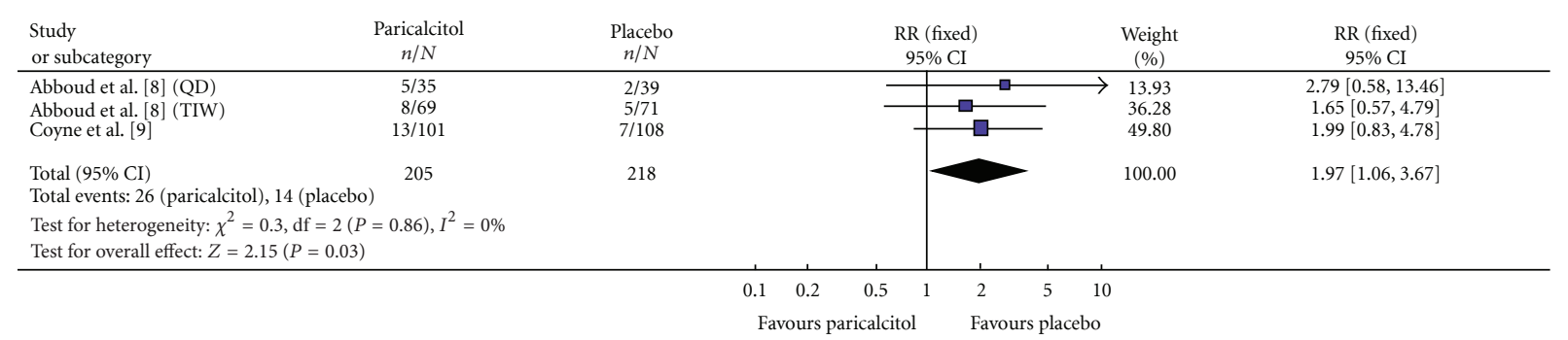

FIGURE 7: Incidence of an elevation in calcium $\times$ phosphorus product.

TABLE 5: Change in calcium $\times$ phosphorus product levels.

\begin{tabular}{lccr}
\hline Reference & Paricalcitol group & Placebo group & $n / N$ \\
& $n / N$ & $2 / 39$ & $P$ value \\
\hline Abboud et al. [8] (QD) & $5 / 35$ & $5 / 71$ & 0.245 \\
Abboud et al. [8] (TIW) & $8 / 69$ & $7 / 108$ & 0.396 \\
Coyne et al. [9] & $13 / 101$ & 0.161 \\
\hline
\end{tabular}

many tissues [36]. Intestinal activation of the VDR can cause hypercalcemia and hyperphosphatemia by enhanced intestinal absorption. Hyperphosphatemia by itself has been associated with increased mortality in patients on dialysis as well as those with CKD not yet on dialysis [36, 37]. Paricalcitol has more selective activation of VDR in the parathyroid gland over that in the intestine and bone and offers the possibility of minimizing the risk of hypercalcemia and hyperphosphatemia, while still significantly reducing PTH $[30,38,39]$.

The goal of the current meta-analysis was to assess whether there is sufficient evidence-based data to recommend Paricalcitol for the management of SHPT in chronic kidney disease patients not yet on dialysis. A second goal of this meta-analysis was to assess whether there is sufficient evidence-based data to recommend Paricalcitol in the management of proteinuric renal disease.

The results of the current meta-analysis indicate that Paricalcitol can decrease iPTH levels significantly with a relatively low incidence of hypercalcemia and hyperphosphatemia. However, we noted that there was a statistically significant difference in elevated $\mathrm{Ca} \times \mathrm{P}$ product levels, which was not found in previous studies [8, 9, 14]. A recently published meta-analysis including patients with CKD and patients with ESRD on dialysis did not identify any safety issues [14]. In contrast, our study looked at patients with CKD only and this safety issue was readily identified. Although there is no statistically significant difference in the incidences of hypercalcemia and hyperphosphatemia between Paricalcitol compared to placebo, an element of caution is needed in interpreting this data. Active vitamin $\mathrm{D}$ analogues, including Paricalcitol, are very likely to have a dose-dependent effect, with higher doses possibly resulting in both hypercalcemia and hyperphosphatemia potentially contributing to an increase in cardiovascular complications. In the studies included in this review, there was insufficient power to identify a dose-response effect. Furthermore, the studies included used $5.5 \mathrm{mg} / \mathrm{dL}$ as the upper limit of the acceptable range for the serum phosphate. This level is recommended in the KDOQI guidelines for patients on dialysis [40]. However, patients with chronic kidney disease usually do not reach such high levels and the current KDIGO guidelines recommend targeting the serum phosphate to the normal range, in this case a level $(<4.0 \mathrm{mg} / \mathrm{dL})$ [24]. Thus, the currently available data may grossly underestimate the effect of Paricalcitol on the serum phosphate in this patient group with CKD not on dialysis.

Paricalcitol has recently been shown to reduce proteinuria in patients with diabetic kidney disease $[4-6,12,14]$. De Zeeuw et al. [12] reported changes in urinary albuminto-creatinine ratio (UACR) and did not note a dose-response relation with Paricalcitol. Due to the limited number of studies included in this meta-analysis about this component, we also did not observe a significant difference between the 1 microgram Paricalcitol treated groups compared with the 2 microgram Paricalcitol treated groups in reducing proteinuria. The current meta-analysis included three studies $[4,5,12]$ that found a significant decrease in proteinuria with Paricalcitol therapy compared with placebo. Agarwal et al. [6] analyzed data from a randomized controlled trial comparing Paricalcitol with placebo for the treatment of secondary hyperparathyroidism in chronic kidney disease. Urinalysis dipstick proteinuria (qualitative) was assessed, and a decrease in proteinuria occurred in $51 \%$ of Paricalcitol-treated patients compared with $25 \%$ of controls. Szeto et al. [13] studied 10 patients with immunoglobulin. A nephropathy-treated with calcitriol, $0.5 \mathrm{ug}$, twice-week for 12 weeks and found a significant decrease in proteinuria. Moreover, in studies [4-6, 12, 13], ACE inhibitors and/or ARBs were used in the majority of patients, and the interaction with a decrease in proteinuria was not significant in the patients receiving ACE inhibitors or ARBs. There may be several possible mechanisms of action for this effect on reducing proteinuria, though none are definitive. Results of experimental 
studies suggest that the reduction in proteinuria induced by Paricalcitol is caused by inhibition of T-cell proliferation and activation $[24,41]$, reduced cytokine and transforming growth factor $\beta$ production, [42] protection of podocytes [43], and suppression of the renin-angiotensin system [44, 45].

The result of changes in eGFR in the current metaanalysis should also be interpreted with caution, because relevant data for this meta-analysis were highly heterogeneous. The studies selected contained descriptive analyses that supported the hypothesis that there was no clinically or statistically significant difference in eGFR between Paricalcitol and placebo groups. This same observation in reference to eGFR was noted in other studies as well $[12-14,46]$.

One of the major limitations of this meta-analysis is the inclusion of only a limited number of studies that met the predetermined set of entry criteria. To minimize bias, we thoroughly carried out searches across different databases using explicit criteria for study selection, data analysis, and data abstraction. Not all of the studies used intention to treat analysis, and allocation concealment was adequate in only five studies. The absence of both of these components could potentially lead to bias. In addition, we could not assess a funnel plot to reveal possible publication bias. Furthermore, the current KDIGO guideline for the target serum phosphorus is a normal level usually defined as $<4.0 \mathrm{mg} / \mathrm{dL}$ [40]. However, each of the studies included in this metaanalysis used a definition of an elevated serum phosphorus as $>5.5 \mathrm{mg} / \mathrm{dL}$. The data to recalculate the effect of Paricalcitol using the currently accepted lower serum phosphorus target was not available to us. Lastly, the small number of studies that addressed the use of Paricalcitol for the treatment of proteinuria in patients without diabetic kidney disease contain insufficient data to conclude that Paricalcitol can decrease proteinuria in these conditions.

In conclusion, there is sufficient evidence based data to conclude that Paricalcitol can effectively decrease iPTH levels in patients with CKD not yet on dialysis. We feel that the data evaluating the effects on serum calcium and phosphate are troublesome and our inability to demonstrate adverse events is limited by insufficient power in the analysis. Moreover, the definition of hyperphosphatemia masked the effect on the serum phosphorus rendering this data largely uninterpretable. The data evaluating the effect on the serum calcium although not statistically significant with a $P<0.09$, definitely showed a trend towards hypercalcemia. This trend was also identified in a previously published meta-analysis which included both patients with CKD and ESRD on dialysis [14]. The statistically significant abnormality highlighting the elevation in $\mathrm{Ca} \times \mathrm{P}$ product supports the notion that clinically significant abnormalities in calcium and phosphate levels may be present with active vitamin $\mathrm{D}$ analogue treatment including Paricalcitol. We recommend caution in the use of vitamin $\mathrm{D}$ analogues including Paricalcitol in the management of SHPT in CKD patients not on dialysis and advise using the lowest effective dose with careful monitoring for the development of hypercalcemia and hyperphosphatemia.

There is also evidence-based data to conclude that Paricalcitol can lead to a significant reduction in proteinuria in patients with diabetic CKD with no apparent measurable impact on kidney function, but not in the case of patients with nondiabetic kidney disease where there is insufficient data. Due to inherent limitations of meta-analysis, larger association studies or multicentric case-control studies are needed to confirm these findings, especially the effect on the serum phosphate using the currently accepted lower target blood level and the effect on the serum calcium using a larger database with sufficient power analysis.

\section{Conflict of Interests}

The authors declare that they have no conflict of interests.

\section{Acknowledgments}

The authors would like to thank the renal group of Tongji Hospital for assistance with the conduct of the study. The authors would also like to thank Drs. Pooneth Alborzi and Dick de Zeeuw for their support during the paper preparation.

\section{References}

[1] M. Mizobuchi, Y. Aoshima, and T. Akizawa, "CKD-MBD (chronic kidney disease-mineral and bone disorder). CKDMBD: chronic kidney disease-mineral and bone disorder," Clinical Calcium, vol. 20, no. 7, pp. 995-1003, 2010.

[2] E. Suchowierska and M. Myśliwiec, "Chronic kidney disease related to mineral and bone disorders," Polski Merkuriusz Lekarski, vol. 28, no. 164, pp. 138-143, 2010.

[3] H. Komaba, "CKD-MBD (Chronic Kidney Disease-Mineral and Bone Disorder). Role of FGF23-Klotho axis in CKD-MBD," Clinical Calcium, vol. 20, no. 7, pp. 1028-1036, 2010.

[4] S. Fishbane, H. Chittineni, M. Packman, P. Dutka, N. Ali, and N. Durie, "Oral paricalcitol in the treatment of patients with CKD and proteinuria: a randomized trial," American Journal of Kidney Diseases, vol. 54, no. 4, pp. 647-652, 2009.

[5] P. Alborzi, N. A. Patel, C. Peterson et al., "Paricalcitol reduces albuminuria and inflammation in chronic kidney disease a randomized double-blind pilot trial," Hypertension, vol. 52, no. 2, pp. 249-255, 2008.

[6] R. Agarwal, M. Acharya, J. Tian et al., "Antiproteinuric effect of oral paricalcitol in chronic kidney disease," Kidney International, vol. 68, no. 6, pp. 2823-2828, 2005.

[7] K. J. Martin, E. A. González, M. Gellens, L. L. Hamm, H. Abboud, and J. Lindberg, "19-nor-1- $\alpha$-25-dihydroxyvitamin D2 (paricalcitol) safely and effectively reduces the levels of intact parathyroid hormone in patients on hemodialysis," Journal of the American Society of Nephrology, vol. 9, no. 8, pp. 1427-1432, 1998.

[8] H. Abboud, D. Coyne, O. Smolenski et al., "A comparison of dosing regimens of paricalcitol capsule for the treatment of secondary hyperparathyroidism in CKD stages 3 and 4," American Journal of Nephrology, vol. 26, no. 1, pp. 105-114, 2006.

[9] D. Coyne, M. Acharya, P. Qiu et al., "Paricalcitol capsule for the treatment of secondary hyperparathyroidism in stages 3 and 4 CKD," American Journal of Kidney Diseases, vol. 47, no. 2, pp. 263-276, 2006. 
[10] L. A. Greenbaum, N. Benador, S. L. Goldstein et al., "Intravenous paricalcitol for treatment of secondary hyperparathyroidism in children on hemodialysis," American Journal of Kidney Diseases, vol. 49, no. 6, pp. 814-823, 2007.

[11] E. A. Ross, J. Tian, H. Abboud et al., "Oral paricalcitol for the treatment of secondary hyperparathyroidism in patients on hemodialysis or peritoneal dialysis," American Journal of Nephrology, vol. 28, no. 1, pp. 97-106, 2007.

[12] D. De Zeeuw, R. Agarwal, M. Amdahl et al., "Selective vitamin $\mathrm{D}$ receptor activation with paricalcitol for reduction of albuminuria in patients with type 2 diabetes (VITAL study): a randomised controlled trial," The Lancet, vol. 376, no. 9752, pp. 1543-1551, 2010.

[13] C. C. Szeto, K. M. Chow, B. C. H. Kwan, K. Y. Chung, C. B. Leung, and P. K. T. Li, "Oral calcitriol for the treatment of persistent proteinuria in immunoglobulin a nephropathy: an uncontrolled trial," American Journal of Kidney Diseases, vol. 51, no. 5, pp. 724-731, 2008.

[14] J. Cheng, W. Zhang, X. Zhang, X. Li, and J. Chen, "Efficacy and safety of paricalcitol therapy for chronic kidney disease: a metaanalysis," Clinical Journal of the American Society of Nephrology, vol. 7, no. 3, pp. 391-400, 2012.

[15] T. Shoji, K. Shinohara, E. Kimoto et al., "Lower risk for cardiovascular mortality in oral $1 \alpha$-hydroxy vitamin $\mathrm{D}_{3}$ users in a haemodialysis population," Nephrology Dialysis Transplantation, vol. 19, no. 1, pp. 179-184, 2004.

[16] M. Teng, M. Wolf, E. Lowrie, N. Ofsthun, J. M. Lazarus, and R. Thadhani, "Survival of patients undergoing hemodialysis with paricalcitol or calcitriol therapy," New England Journal of Medicine, vol. 349, no. 5, pp. 446-456, 2003.

[17] F. Tentori, W. C. Hunt, C. A. Stidley et al., "Mortality risk among hemodialysis patients receiving different vitamin D analogs," Kidney International, vol. 70, no. 10, pp. 1858-1865, 2006.

[18] S. Mathew, R. J. Lund, L. R. Chaudhary, T. Geurs, and K. A. Hruska, "Vitamin D receptor activators can protect against vascular calcification," Journal of the American Society of Nephrology, vol. 19, no. 8, pp. 1509-1519, 2008.

[19] A. R. Jadad, R. A. Moore, D. Carroll et al., "Assessing the quality of reports of randomized clinical trials: is blinding necessary?" Controlled Clinical Trials, vol. 17, no. 1, pp. 1-12, 1996.

[20] J. P. Higgins and S. Green, Cochrane Handbook for Systematic Reviews of Interventions, Cochrane Collaboration, 2008.

[21] J. P. T. Higgins, S. G. Thompson, J. J. Deeks, and D. G. Altman, "Measuring inconsistency in meta-analyses," British Medical Journal, vol. 327, no. 7414, pp. 557-560, 2003.

[22] J. P. T. Higgins and S. G. Thompson, "Quantifying heterogeneity in a meta-analysis," Statistics in Medicine, vol. 21, no. 11, pp. 1539-1558, 2002.

[23] H. J. Lambers Heerspink, R. Agarwal, D. W. Coyne et al., "The selective vitamin d receptor activator for albuminuria lowering (VITAL) study: study design and baseline characteristics," American Journal of Nephrology, vol. 30, no. 3, pp. 280-286, 2009.

[24] Kidney Disease: Improving Global Outcomes (KDIGO) CKDMBD Work Group, "KDIGO clinical practice guideline for the diagnosis, evaluation, prevention, and treatment of chronic kidney disease-mineral and bone disorder (CKD-MBD)," Kidney International, Supplement, vol. 76, no. 113, pp. S1-S130, 2009.

[25] M. Cozzolino, E. Missaglia, A. Ortiz et al., "Vascular calcification in chronic kidney disease," Recenti Progressi in Medicina, vol. 101, no. 11, pp. 442-452, 2010.
[26] N. Joki and Y. Tanaka, “CKD-MBD (Chronic Kidney DiseaseMineral and Bone Disorder). Atheroma and vascular calcification in hemodialysis patients," Clinical Calcium, vol. 20, no. 7, pp. 1061-1066, 2010.

[27] J. Bover, A. Aguilar, J. P. Baas et al., "Calcimimetics in the chronic kidney disease-mineral and bone disorder," International Journal of Artificial Organs, vol. 32, no. 2, pp. 108-121, 2009.

[28] W. G. Goodman, "Recent developments in the management of secondary hyperparathyroidism," Kidney International, vol. 59, no. 3, pp. 1187-1201, 2001.

[29] E. Slatopolsky, C. Weerts, and J. Thielen, "Marked suppression of secondary hyperparathyroidism by intravenous administration of 1,25-dihydroxycholecalciferol in uremic patients," Journal of Clinical Investigation, vol. 74, no. 6, pp. 2136-2143, 1984.

[30] A. J. Brown, J. Finch, and E. Slatopolsky, "Differential effects of 19-nor-1,25-dihydroxyvitamin D2 and 1,25-dihydroxyvitamin $\mathrm{D}_{3}$ on intestinal calcium and phosphate transport," Journal of Laboratory and Clinical Medicine, vol. 139, no. 5, pp. 279-284, 2002.

[31] R. J. Lund, D. L. Andress, M. Amdahl, L. A. Williams, and R. P. Heaney, "Differential effects of paricalcitol and calcitriol on intestinal calcium absorption in hemodialysis patients," American Journal of Nephrology, vol. 31, no. 2, pp. 165-170, 2010.

[32] J. Silver, J. Russell, and L. M. Sherwood, "Regulation by vitamin D metabolites of messenger ribonucleic acid for preproparathyroid hormone in isolated bovine parathyroid cells," Proceedings of the National Academy of Sciences of the United States of America, vol. 82, no. 12, pp. 4270-4273, 1985.

[33] J. Russell, D. Lettieri, and L. M. Sherwood, "Suppression by $1,25(\mathrm{OH})_{2} \mathrm{D}_{3}$ of transcription of the pre-proparathyroid hormone gene," Endocrinology, vol. 119, no. 6, pp. 2864-2866, 1986.

[34] J. Silver, T. Naveh-Many, and H. Mayer, "Regulation by vitamin $\mathrm{D}$ metabolites of parathyroid hormone gene transcription in vivo in the rat," Journal of Clinical Investigation, vol. 78, no. 5, pp. 1296-1301, 1986.

[35] T. Okazaki, T. Igarashi, and H. M. Kronenberg, " 5 '-flanking region of the parathyroid hormone gene mediates negative regulation by $1,25-(\mathrm{OH})_{2}$ vitamin $\mathrm{D}_{3}$," Journal of Biological Chemistry, vol. 263, no. 5, pp. 2203-2208, 1988.

[36] D. Gupta, S. Brietzke, M. R. Hayden, L. R. Kurukulasuriya, and J. R. Sowers, "Phosphate metabolism in cardiorenal metabolic disease," Cardiorenal Medicine, vol. 1, no. 4, pp. 261-270, 2011.

[37] J. Kendrick and M. Chonchol, "The role of phosphorus in the development and progression of vascular calcification," American Journal of Kidney Diseases, vol. 58, no. 5, pp. 826-834, 2011.

[38] M. Teng, M. Wolf, E. Lowrie, N. Ofsthun, J. M. Lazarus, and R. Thadhani, "Survival of patients undergoing hemodialysis with paricalcitol or calcitriol therapy," New England Journal of Medicine, vol. 349, no. 5, pp. 446-456, 2003.

[39] D. W. Coyne, M. Grieff, S. N. Ahya, K. Giles, K. Norwood, and E. Slatopolsky, "Differential effects of acute administration of 19-nor-1,25-dihydroxy-vitamin D2 and 1,25-dihydroxyvitamin $\mathrm{D}_{3}$ on serum calcium and phosphorus in hemodialysis patients," American Journal of Kidney Diseases, vol. 40, no. 6, pp. 1283-1288, 2002. 
[40] National Kidney Foundation, "KDOQI clinical practice guidelines for bone metabolism and disease in chronic kidney disease," American Journal of Kidney Diseases, vol. 42, supplement 3, pp. S1-S201, 2003.

[41] A. K. Bhalla, E. P. Amento, and T. L. Clemens, "Specific high-affinity receptors for 1,25-dihydroxyvitamin $\mathrm{D}_{3}$ in human peripheral blood mononuclear cells: presence in monocytes and induction in T lymphocytes following activation," Journal of Clinical Endocrinology and Metabolism, vol. 57, no. 6, pp. 1308-1310, 1983.

[42] L. Helming, J. Böse, J. Ehrchen et al., “ $1 \alpha$,25-dihydroxyvitamin $\mathrm{D}_{3}$ is a potent suppressor of interferon $\gamma$-mediated macrophage activation," Blood, vol. 106, no. 13, pp. 4351-4358, 2005.

[43] G. Penna and L. Adorini, " $1 \alpha, 25$-dihydroxyvitamin $\mathrm{D}_{3}$ inhibits differentiation, maturation, activation, and survival of dendritic cells leading to impaired alloreactive T cell activation," Journal of Immunology, vol. 164, no. 5, pp. 2405-2411, 2000.

[44] Y. C. Li, J. Kong, M. Wei, Z. F. Chen, S. Q. Liu, and L. P. Cao, "1,25-Dihydroxyvitamin $\mathrm{D}_{3}$ is a negative endocrine regulator of the renin-angiotensin system," Journal of Clinical Investigation, vol. 110, no. 2, pp. 229-238, 2002.

[45] Z. Zhang, Y. Zhang, G. Ning, D. K. Deb, J. Kong, and C. L. Yan, "Combination therapy with AT1 blocker and vitamin D analog markedly ameliorates diabetic nephropathy: blockade of compensatory renin increase," Proceedings of the National Academy of Sciences of the United States of America, vol. 105, no. 41, pp. 15896-15901, 2008.

[46] E. Slatopolsky, A. Brown, and A. Dusso, "Pathogenesis of secondary hyperparathyroidism," Kidney International, Supplement, vol. 56, pp. S14-S19, 1999. 


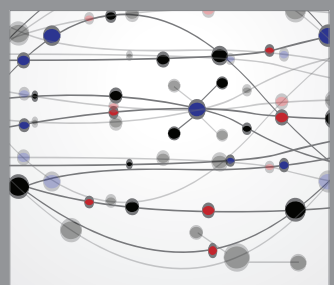

The Scientific World Journal
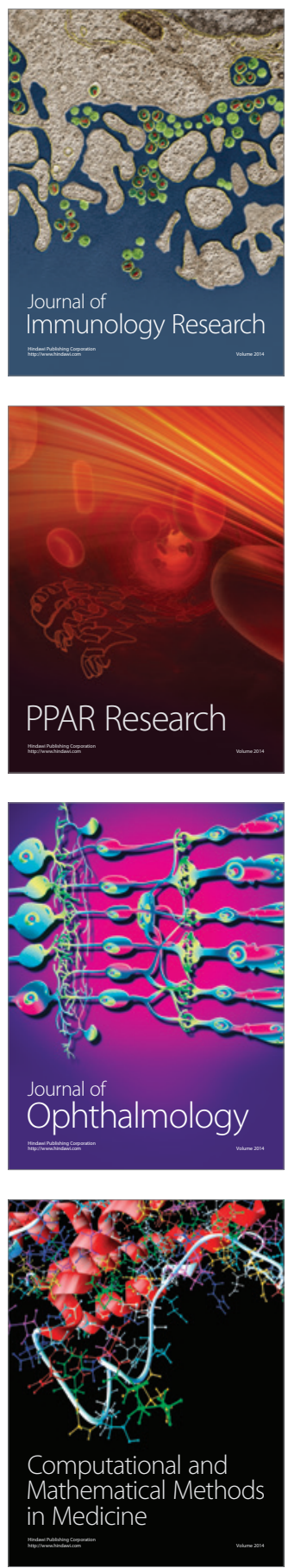

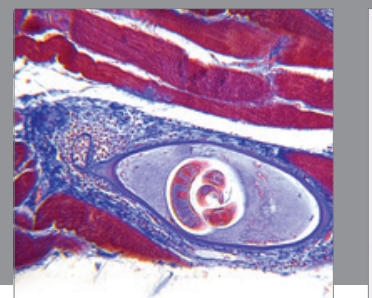

Gastroenterology

Research and Practice
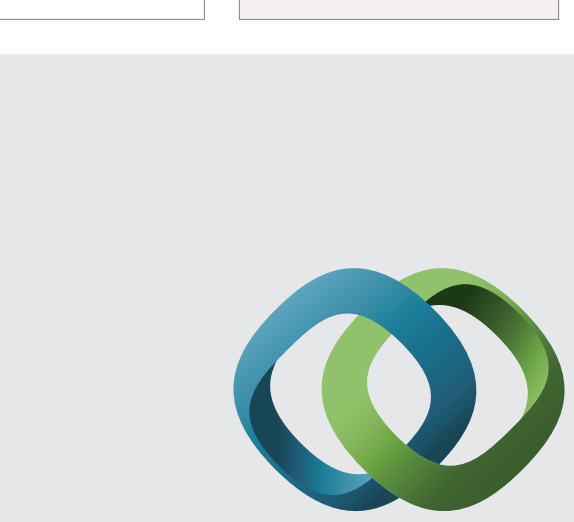

\section{Hindawi}

Submit your manuscripts at

http://www.hindawi.com
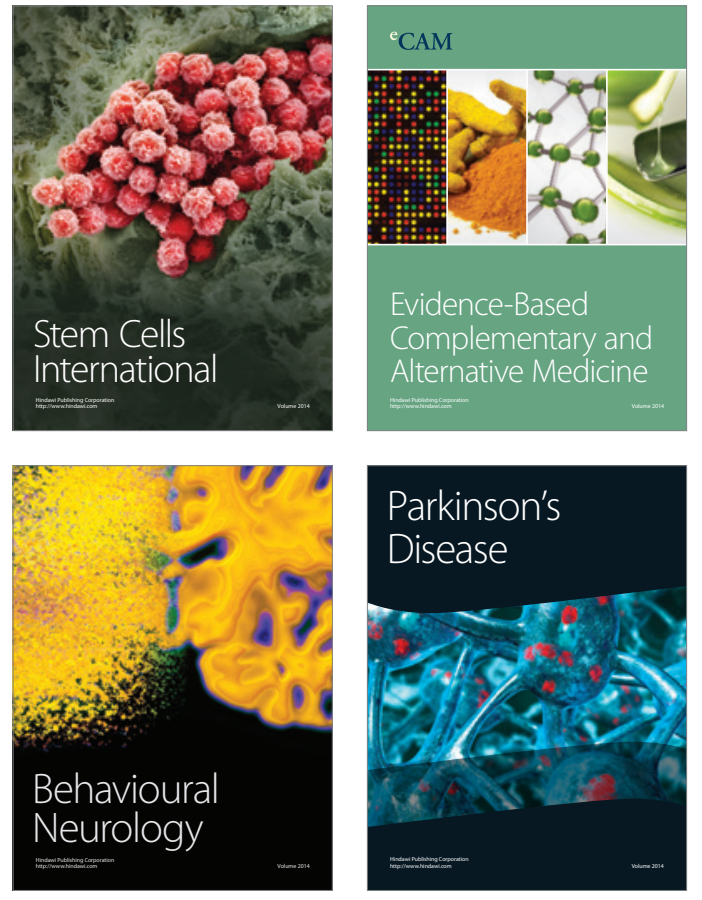
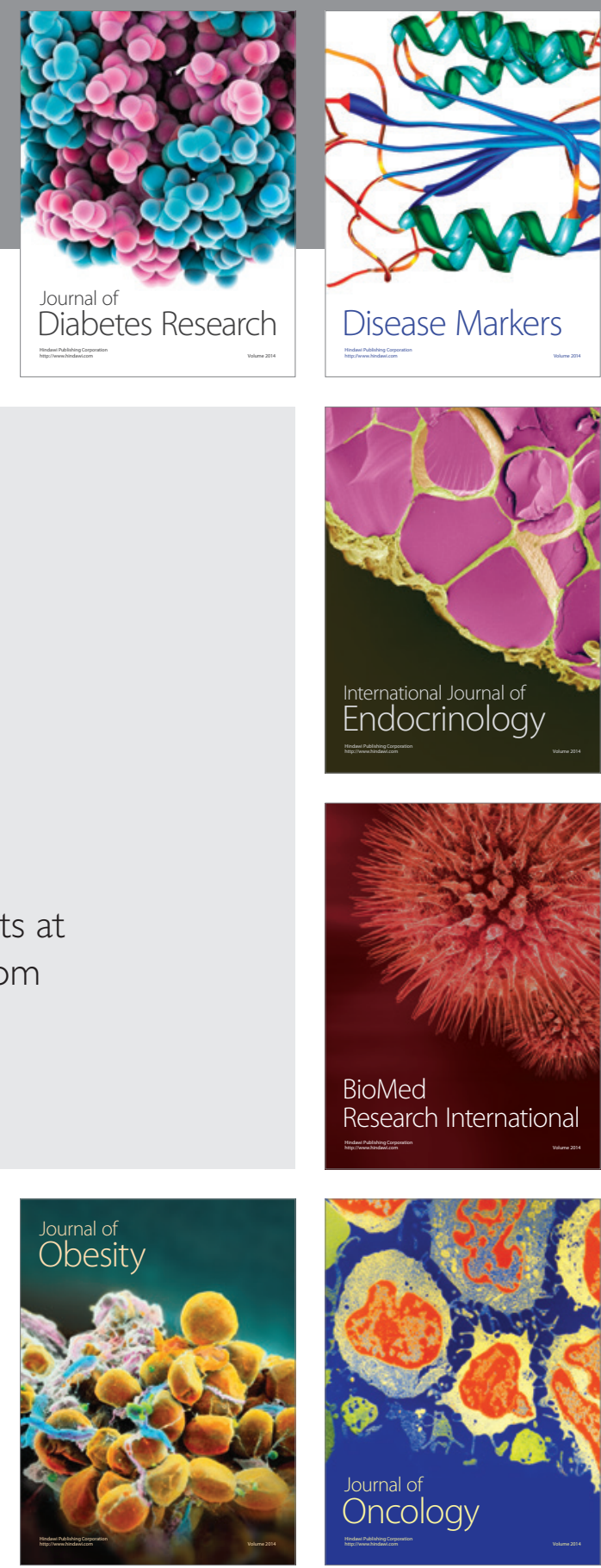

Disease Markers
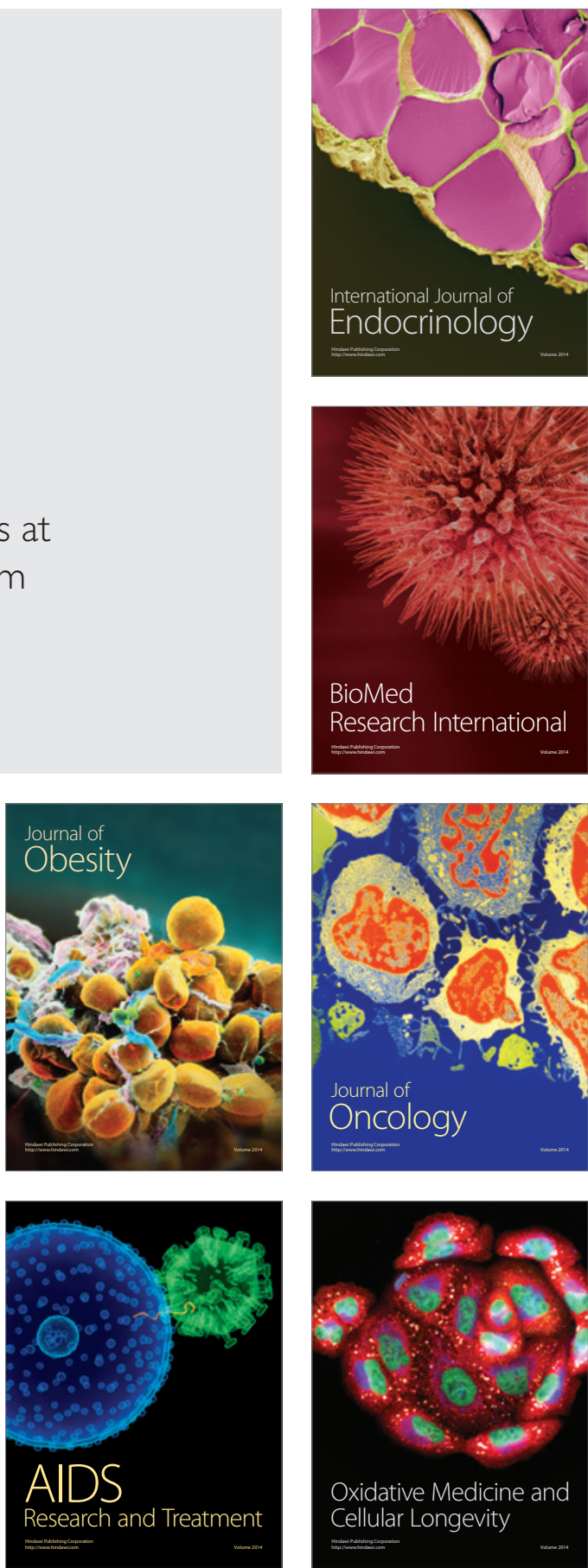